Pathologe 2011 - [Suppl 2] 32:376-377

DOI 10.1007/s00292-011-1464-9

Online publiziert: 31. Juli 2011

(c) Springer-Verlag 2011
W. Feiden
MVZ für Histologie, Zytologie und Molekulare Diagnostik, Trier

\section{Parviz Mehraein}

\subsubsection{1 - 16.10.2009}

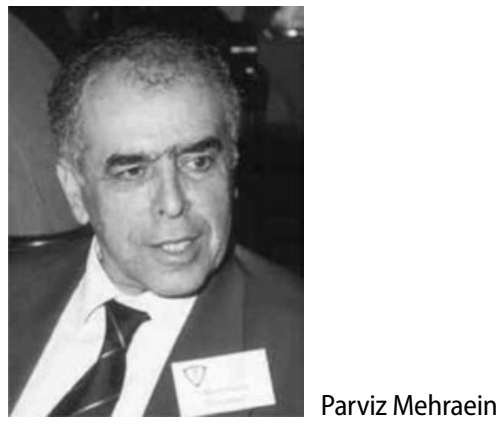

Am 16.10.2009 verstarb kurz vor Vollendung seines 78. Lebensjahres Parviz Mehraein, ordentlicher Professor für Neuropathologie und ehemaliger Vorstand des Instituts für Neuropathologie der Ludwig-Maximilians-Universität (LMU) in München. Die Anzeige seines Todes trug den Satz: „Wir sind alle Reisende, welch Wunder, daß die Karawane den beweint, der das Ziel erreicht hat."

Dieser Satz kennzeichnet den Verstorbenen in mehrerer Hinsicht. Zunächst äußert sich darin sein eher zurückhaltendes, bescheidenes Wesen, aber er hat auch ein Ziel vor Augen - wenn der Weg dahin auch durchaus lang und beschwerlich ist und man einen langen Atem braucht. Mehraein drängte sich nie in den Vordergrund, aber jeder unter seinen Fachkollegen kannte und schätzte ihn - sowie darüber hinaus viele Vertreter benachbarter Disziplinen, die in seinem akademischen Leben irgendwann einmal seinen Weg gekreuzt hatten und mit denen er kollegial kooperierte: Neurologen und Psychiater, Pädiater und Humangenetiker, Physiologen, Neuroanatomen und Neurochirurgen und auch Pathologen, sei es im ehemaligen Max-Planck-Institut für Psychiatrie in München-Schwabing, in Hannover oder später wieder in München, in seiner Zeit als Vorstand des Instituts für Neuropathologie.

Teil einer Karawane zu sein bedeutet auch, voranzukommen, etwas zu bewegen oder im Fluss zu halten. Mehraein gehörte zu jenen Hochschullehrern, die mehr als andere in seiner Generation junge Neuropathologen habilitiert und sozusagen auf den akademischen Weg gebracht haben und zwar ganz ohne „Schule“ und ohne die Mirabilia einer „Kaderschmiede“. Das war seine Art nicht, sondern eher das wohlwollend unterstützende Begleiten und Beraten „seiner (akademischen) Kinder", von denen alle sozusagen ihre Reise machten, zum Teil in ganz verschiedene Richtungen.

Der vieldeutige Satz von der Karawane weist aber auch auf die Herkunft und den geistigen Hintergrund von Parviz Mehraein hin, denn er stammt aus Mechhed, Iran. Persische Geschichten und Verse, insbesondere von Saadi und Hafis, waren seine stille Leidenschaft. $\mathrm{Zu}$ mancher eher glücklichen Stunde, zu fröhlichen Anlässen oder auch bei weniger erbaulichen oder besorgten Begebenheiten konnte der viel Belesene und auch selbst Schreibende einen treffenden und zumeist tiefgründigen persischen Satz auswendig zitieren - natürlich in seiner Muttersprache und sodann in einer trefflichen, ausgefeilten Übersetzung ins Deutsche.

Bis 1952 hat Mehraein in seiner persischen Geburtsstadt gelebt. Sein Vater war Offizier beim Militär, aber davon hat man Parviz Mehraein wirklich nichts angemerkt, jedenfalls nicht der Unterzeichnete. Nach dem Besuch der Grundund Oberschule von 1938 bis 1950 hat Mehraein in seiner Heimatstadt das Medizinstudium begonnen (1950) und dieses von 1952 bis 1958 - nach der Übersiedlung in die BRD - fortgesetzt: zunächst in Freiburg (bis 1955) - hier zusätzlich kombiniert mit dem Studium der Psychologie - sowie danach an der LMU, wo er 1958 das medizinische Staatsexamen erwarb. Das Thema der Dissertationsschrift lautete: „Epileptische Wesensänderung und EEG-Befunde“; Doktorvater war Prof. Dr. Joachim Ernst Meyer, Nervenklinik der Universität München. Nach der Medizinalassistentenzeit trat Mehraein 1962 als wissenschaftlicher Assistent in das von Gerd Peters geleitete Max-Planck-Institut für Psychiatrie in München ein, und zwar in die Abteilung Neuropathologie. Diese Abteilung unter Gerd Peters war damals in der Tat eine "Schule“, noch ganz im Geiste von Spielmeyer und unter persönlicher Anwesenheit des vormaligen Direktors Willibald Scholz, eine "Schule“, aus der mehrere Wissenschaftler als Lehrstuhlinhaber für das sich damals universitär ausbreitende Fach Neuropathologie berufen wurden - und nicht nur Neuropathologen. Auch Parviz Mehraein gehörte später dazu. Zunächst aber war Mehraein in dieser „Hochburg der Hirnforschung" damit, nämlich mit „klassischer" Neuropathologie beschäftigt, also Makroskopie und Mikroskopie von Gehirnen mit ganz verschiedenen Krankheitsprozessen. Entsprechend vielfältig waren die Themen der Publikationen aus dieser Zeit, auch unter Einschluss tierexperimenteller Hirnbefunde. Mehraein wurde Oberassistent und Arbeitsgruppenleiter der Neuropathologie. Viele der langwährenden und vielfach freundschaftlichen Kontakte resultierten aus jener Zeit in Schwabing, zumal zu den damals dort arbeitenden japanischen Kollegen. Die Arbeit im „MPI“ gipfelte in der 
Habilitationsschrift mit einem Thema zu morphometrischen Untersuchungen bei Morbus Alzheimer und seniler Demenz. Mehraein wies auf die Bedeutung der Synapsenreduktion hin, ein Befund, der in der heutigen Forschung dieser nach wie vor sehr aktuellen degenerativen Hirnerkrankung etwas in den Hintergrund geraten ist. Erfreulich, dass im Zentrum für Neuropathologie und Prionforschung, wie sich das Institut für Neuropathologie der LMU seit geraumer Zeit benennt, dieses Thema mit „High-tech-Methoden“ erfolgreich bearbeitet wird. Der Habilitation an der LMU in München am 12.01.1978 sozusagen als „Externer“ - folgte rasch ein Ruf an die damals neu geschaffene Professur für Neuropathologie an der Medizinischen Hochschule Hannover (MHH). Von 1978 bis 1983 war Mehraein Direktor des Instituts für Neuropathologie der MHH. Er hat in Hannover das Fach etabliert und die Abteilung quasi von den Grundmauern aus aufgebaut.

1983 kam Mehraein nach München zurück, nachdem er den ehrenvollen Ruf auf den Lehrstuhl für Neuropathologie an der LMU erhalten hatte und damit die Nachfolge von Otto Stochdorph antrat. Diese Münchner Zeit war eine außerordentlich fruchtbare, wenngleich zu Beginn überschattet von einem tragischen tödlichen Unfall einer seiner vier geliebten Töchter, ein Schicksalsschlag, der bis zu seinem Lebensende an ihm nagte, auch wenn er es sich kaum anmerken ließ.

In der Münchner Zeit wurde das Institut für Neuropathologie, damals noch beengt untergebracht in dem mächtigen Pathologiebau an der Thalkirchner Straße, systematisch ausgebaut. Dabei war ein Meilenstein die Einrichtung des Referenzzentrums für Neurodegenerative Erkrankungen des Nervensystems der Deutschen Gesellschaft für Neuropathologie und Neuroanatomie (DGNN) im Jahr 1995. Mehraein leitete dieses Referenzzentrum, und außerdem kommissarisch das Universitätsinstitut über 3 Jahre lang, bis 200o. In die Münchner Zeit fielen die Habilitationen von Neuropathologen, die unter seiner Betreuung und in der offenen, förderlichen Atmosphäre des Instituts abgeschlossen wurden. Alle seine akademischen Kinder gingen auf die Reise: D. Stavrou - primär aus der Veteri-
när-(Neuro-)Pathologie gekommen, später Professor für Neuropathologie und Direktor des Instituts am Universitätsklinikum Hamburg-Eppendorf, W. Feiden Universität des Saarlandes, H. Kretzschmar - sein Nachfolger im Amt und derzeitiger Direktor des Zentrums für Neuropathologie und Prionforschung der LMU, M. Graeber - Neuroscience Center, King Fahad Medical City, Riyadh/Saudi-Arabien, S. Weis - Pathologie, Nervenklinik Wagner-Jauregg, Linz/Österreich, S. Kösel und E. Grasbon-Frödel, beide München. R. Egensperger - Neuropathologie LMU München - kann getrost dazu gezählt werden, wenngleich die Habilitation unter G. Walter, Mehraeins Nachfolger an der $\mathrm{MHH}$, über die Bühne ging.

Im Jahr 1994 richtete Mehraein als Vorsitzender der DGNN die Jahrestagung der Gesellschaft in München aus, wo man schon 1982 (O. Stochdorph) und 1990 (E. Dahme) zusammengekommen war. Der 70. Geburtstag wurde 2001 auch mit einer würdigen akademischen Feier begangen, mit wissenschaftlichen Vorträgen seiner ehemaligen Mitarbeiter. Die Jahre danach, sozusagen im Ruhestand, wurden in der Tat dann etwas ruhiger und privater, leider überschattet von einer malignen Tumorerkrankung, die sich zunächst relativ „ruhig“ verhielt, am Ende aber doch aggressiver wurde. Unter Schmerzen kam Parviz Mehraein zum Ziel seiner Reise. Die Bestattung seines Leichnams fand im engsten familiären Kreis statt. Seine „akademischen Kinder“ - und nicht nur die werden Parviz Mehraein, in Hochachtung vor dem Menschen und dem akademischen Lehrer, in dankbarer Erinnerung behalten.

\section{Wolfgang Feiden, Trier}

\section{Korrespondenzadresse \\ Prof. Dr. W. Feiden \\ MVZ für Histologie, \\ Zytologie und Molekulare Diagnostik \\ Max-Planck-Str. 18+20, 54296 Trier \\ neuropathologie@hotmail.de}

